\title{
Comprehensive Phylogenetic Analysis of Root-knot Nematodes Predicts Emerging Virulent Species
}

\author{
Kamrul Islam ${ }^{1}$, *, Mohammad Jakir Hosen ${ }^{1}$, Sourav Chakraborty ${ }^{1}$, Auditi Purkaystha ${ }^{1}$, \\ Mahmudul Hasan ${ }^{1,2}$, Bonhi Elora ${ }^{3}$ \\ ${ }^{1}$ Department of Genetic Engineering and Biotechnology, Shahjalal University of Science and Technology, Sylhet, Bangladesh \\ ${ }^{2}$ Department of Pharmaceuticals and Industrial Biotechnology, Sylhet Agricultural University, Sylhet, Bangladesh \\ ${ }^{3}$ Anandaniketan, Sylhet, Bangladesh
}

Email address:

kamrul-gen@sust.edu (K. Islam)

${ }^{*}$ Corresponding author

\section{To cite this article:}

Kamrul Islam, Mohammad Jakir Hosen, Sourav Chakraborty, Auditi Purkaystha, Mahmudul Hasan, Bonhi Elora. Comprehensive Phylogenetic Analysis of Root-knot Nematodes Predicts Emerging Virulent Species. Computational Biology and Bioinformatics. Vol. 8, No. 1, 2020, pp. 1-8. doi: 10.11648/j.cbb.20200801.11

Received: September 17, 2019; Accepted: December 30, 2019; Published: February 3, 2020

\begin{abstract}
Among the root-knot nematodes three Meloidogyne species namely Meloidogyne incognita, M. javanica, and M. arenaria are emerging as an important pest of many cultivated plants, and recognized as the most economically destructive plant parasitic nematodes species of all over the world. Although other root-knot nematodes may virulent for plant but limited information is available. Thus, a comprehensive bioinformatics analysis including sequence acquisition, multiple sequence alignment and the phylogenetic tree construction for well-known Meloidogyne species was employed to predict the emerging virulent species. About eighty seven (87) $18 \mathrm{~S}$ rRNA sequences of three damaging Meloidogyne species (M. javanica, M. arenaria and $M$. incognita) were retrieved from NCBI database, and allowed to construct phylogenetic trees using both NJ and ME methods of Molecular Evolution Genetic Analysis (MEGA) tools. Phylogeny analysis revealed that M. enterolobii_1, M. $s p .{ }_{-} i_{-} c 3 a, M . s p \_M j \_c l a$ and $M .{ }_{-} s p .{ }_{-} M j_{-} c 3 a$ are genetically as well as evolutionally related to existing well recognized virulent nematodes. Moreover, evolutionally emerging strains of existing virulent species of M. javanica, M. arenaria and $M$. incognita along with the predicted virulence nematodes could become a great challenge to agriculture. The study could initiate the further analysis for novel insights in the pathogenesis of emerging virulence species of Meloidogyne that must be needed for future crop management strategies.
\end{abstract}

Keywords: Meloidogyne, Virulence, Phylogenetic Analysis, Plant Parasite, 18S rRNA

\section{Introduction}

Root-knot nematodes (RKN) are belong to Meloidogyne genus, are microscopic obligate endo-parasites that live in soil. RKN feed on the roots of a wide range of plant species [1-3] and consider as an important pathogen of numerous plants including food crops, and known as the most economically destructive genus of plant parasitic nematodes in the world $[4,5]$. RKN produce galls on roots that eventually lead to reduced water uptake to shoots that causes yield loss. The severity of yield loss can range from minimal to total depending on the infesting RKN species and crop variety, season, soil type and use of crop rotation [6-9].
Distribution of Meloidogyne species is depends on the ability of these obligate root parasites to become established during overwinter in a geographic area. Among the reported 90 Meloidogyne species, M. incognita, M. javanica, and $M$. arenaria are most damaging [10-12].

Two genomic regions that have been used regularly to characterize species among the nematode taxa are the ribosomal RNA array and the mitochondrial genome. Remarkably, the ribosomal RNA (rRNA) genes and their intervening sequences are the best characterized genes or gene regions in Nematoda [13-15]. Typically, the rRNA array consists of three ribosomal genes including $18 \mathrm{~S}, 5.8 \mathrm{~S}$, and 28S. Recent findings suggest that 14 Meloidogyne species in 
a phylogenetic comparison using nearly complete $18 \mathrm{~S}$ rDNA sequence and reported that within the genus there was about $10 \%$ divergence in pairwise comparisons among species, corresponding to considerable diversity for a relatively conserved gene [16-20]. Recent findings suggested that the sequences of a $18 \mathrm{~S}$ rRNA can also distinguish between species in a genus [21-23]. Thus, polymorphic sites in the $18 \mathrm{~S}$ gene can be exploited to diagnose individual species as well as can give insights of the inter- and intra-species relationship. In addition, molecular diagnosis by using $18 \mathrm{~S}$ rRNA sequence will help to identify and address unforeseen emerging parasites threat to agricultural crop [24, 25]. Although there is no such data is available for Meloidogyne spp. Thus, the present study aims to identify the noxious Meloidogyne spp. based on the comparison with three damaging Meloidogyne species (M. javanica, M. arenariaand $M$. incognita) using $18 \mathrm{~S}$ rRNA through comparing conserve regions and phylogenetic tree construction.

Table 1. Meloidogyne species and the area they showed prevalence in crops.

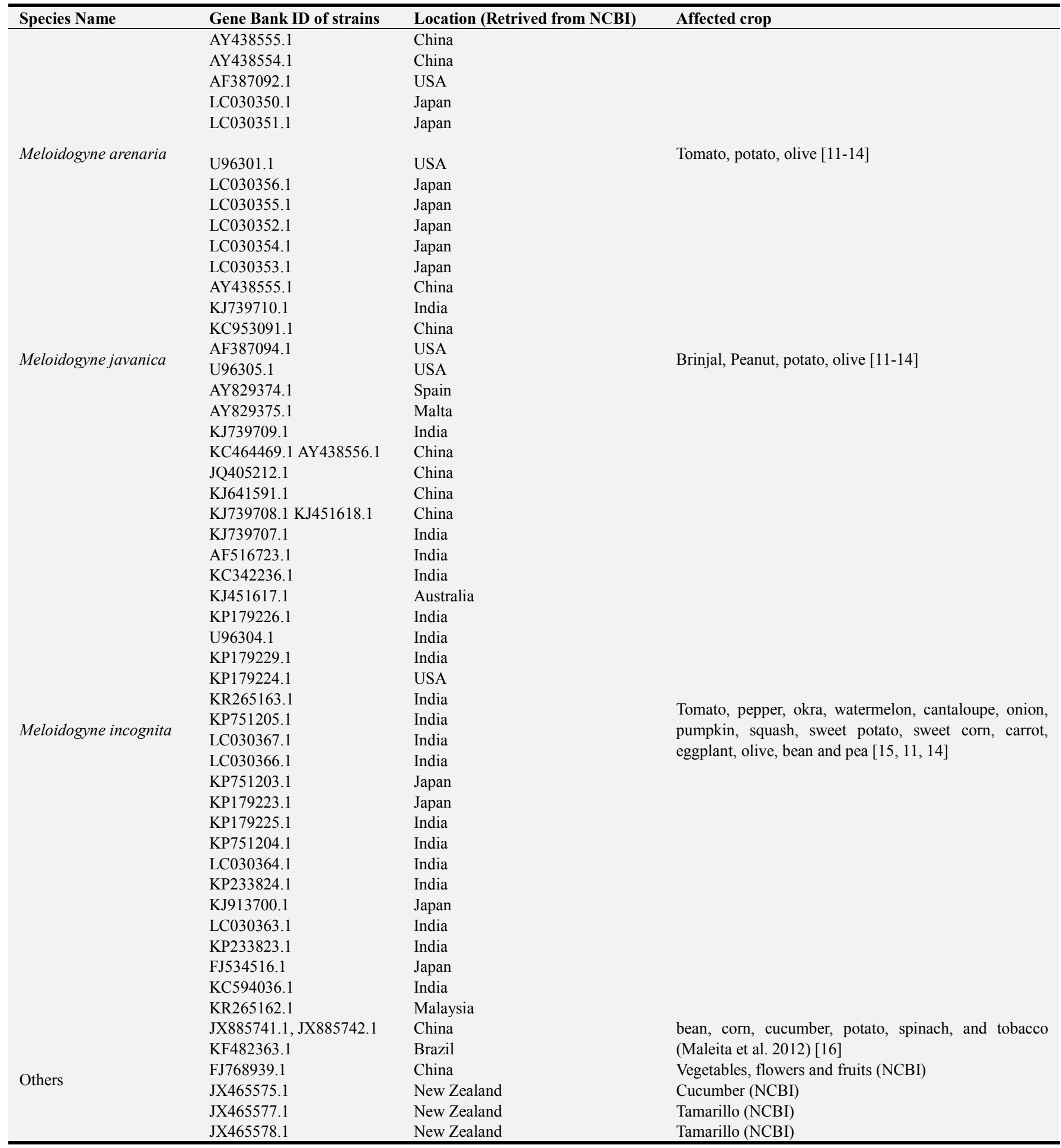




\begin{tabular}{|c|c|c|c|}
\hline Species Name & Gene Bank ID of strains & Location (Retrived from NCBI) & Affected crop \\
\hline & JX465572.1 & New Zealand & Tamarillo (NCBI) \\
\hline & JX465571.1 & New Zealand & Tamarillo (NCBI) \\
\hline & JX465576.1 & New Zealand & Tamarillo (NCBI) \\
\hline & JX465574.1 & New Zealand & Tamarillo (NCBI) \\
\hline & JX465573.1 & New Zealand & Tamarillo (NCBI) \\
\hline & JX465569.1 & New Zealand & Tamarillo (NCBI) \\
\hline & JX465570.1 & New Zealand & Kiwifruit (NCBI) \\
\hline & KF482368.1 & Brazil & Kiwifruit (NCBI) \\
\hline & KF418368.1 & China & Vegetables, flowers and fruits (NCBI) \\
\hline & KF482366.1 & Brazil & Vegetables (NCBI) \\
\hline & JX465565.1 & New Zealand & Vegetables, flowers and fruits (NCBI) \\
\hline & JX465566.1 & New Zealand & Tamarillo (NCBI) \\
\hline & JX465564.1 & New Zealand & Tamarillo (NCBI) \\
\hline & JX465567.1 & New Zealand & Tamarillo (NCBI) \\
\hline & JX465568.1 & New Zealand & Tamarillo (NCBI) \\
\hline & JX465562.1 & New Zealand & Kiwifruit (NCBI) \\
\hline & JX465561.1 & New Zealand & Tamarillo (NCBI) \\
\hline & JF309157.1 & Costa Rica & Kiwifruit (NCBI) \\
\hline & JF309154.1 & Costa Rica & Guava (NCBI) \\
\hline & JF309155.1 & Costa Rica & Malpighia sp. (NCBI) \\
\hline & JF309156.1 & Costa Rica & Guava (NCBI) \\
\hline & JX024149.1 & China & Guava (NCBI) \\
\hline & KT354573.1 & China & Euphorbia tirucalli (NCBI) \\
\hline & KJ146863.1 & China & Banana (NCBI) \\
\hline & KT354575.1 & China & Carrot (NCBI) \\
\hline & KT354565.1 & China & Banana (NCBI) \\
\hline & KP411227.1 & Taiwan & Banana (NCBI) \\
\hline & KF418370.1 & China & Guava (NCBI) \\
\hline & KF418369.1 & China & Vegetables (NCBI) \\
\hline & JQ082448.1 & China & Vegetables (NCBI) Carrot [17] \\
\hline
\end{tabular}

\section{Methods and Materials}

\subsection{Sequence Retrieval}

18S rRNA sequences of three major Meloidogyne species (M. javanica, M. arenaria, and M. incognita) were retrieved from the National Centre for Biotechnology Information database (NCBI) (http://www.ncbi.nlm.nih.gov). Obtained sequences were blasted in the NCBI database (using nucleotide blast query) that revealed total 87 sequences of different Meloidogyne species, which were further used for phylogenetic analysis (Table 1).

\subsection{Multiple Sequence Alignment}

All the retrieved 87 sequences of $18 \mathrm{~s}$ rRNA were aligned using Clustal omega (http://www.ebi.ac.uk/Tools/msa/clustalo/); which can align virtually any number of protein sequences quickly and that delivers accurate alignments [26-28]. Clustal omega 18s rRNA sequence alignment of three major Meloidogyne species (M. javanica, M. arenaria, and M. incognita) and predicted emerging species were also used for the analysis of conserve regions.

\subsection{Phylogenetic Tree Construction}

First, 18S rRNA sequences of these 87 Meloidogyne species were aligned with ClustalW using the default parameters for gap opening and gap extension penalties. The software "Molecular Evolution Genetic Analysis (MEGA)", version 6 [29] was used for phylogenetic analysis. "Neighbour Joining (NJ)" and "Minimum Evolution (ME)" methods were used to construct two phylogenetic tree. Evolutionary distances between species were computed using the "Maximum Composite Likelihood" method [30] and the units of the number of base substitutions per site. Total 1000 replicates of bootstrapping were calculated in this purpose.

\section{Results and Discussion}

Multiple Sequence Alignment obtained from Clustal omega revealed that maximum similarity of Meloidogyne incognita, $M$. javanica, and $M$. arenaria was found with M. enterolobii_1, $M$. sp._Mi_c3a, M. sp_Mj_cla and M._sp._Mj_c3a strains (Figure 1). The phylogenetic trees of $18 \mathrm{~S}$ rDNA sequences from 87 Meloidogyne species that were constructed using both $\mathrm{NJ}$ (Figure 2) and ME (Figure 3) methods were grouped into 7 major clades. Clade- I of Minimum Evolution Phylogenetic Tree (MEPT) differentiated in to 2 sub-clades; the first sub-clade was consist of eight nematodes species including $M$. incognita_1 (Figure 2) and $M$. incognita [31-33], are evolutionally closely related with other Meloidogyne spp. denoted by accession number JF309155.1, FJ534516.1, JF309158.1, JF309154.1, KF418368.1, JF309157.1, JF309156.1 respectively. In case of sub-clade-2, nematode species denoted with the accession number KF482366.1, KF482368.1, JX885742.1 were found to distantly related with the virulent $M$. incognita_1. Interestingly, Clade-I of Neighbor Joining Phylogenetic Tree (NJPT) also shared the same result. In other hand, Clade-II constructed by both MEPT and NJPT comprised of different strains of $M$. 
enterolobii represented by the accession number KF418370.1, JX024149.1， KT354573.1， KP411227.1， KJ146863.1, KF418369.1， KT354565.1， JQ082448.1， KT354575.1 respectively. Evolutionary tree of Clade-II revealed that these $M$. Enterolobii strains are derived from the same ancestor as of $M$. incognita. Among the four strains (LC030363.1, LC030364.1, LC030367.1, LC030366.1) of $M$. incognita derived from the clade-III; LC030363.1, LC030364.1 were closely related and ancestral to two closely related strains (LC030366.1, LC030367.1). A harmonious genetic relationship was also obtained from the clade-III of NJPT. Clade-IV of NJPT showed that two strains of M. arenaria (LC030353.1, LC030356.1) and one strain of $M$. incognita (KP179225.1) were distantly related to each other. In addition, Clade-IV also showed that Meloidogyne sp Mh_c4b (JX465568.1) and Meloidogyne $s p \_M i \_c 2 b(\mathrm{JX} 465567.1)$ are closely related. Clade-IV of
MEPT revealed that $M$. arenaria_1 (LC030353.1) was ancestral to LC030356.1, KP179225.1, JX465568.1, JX465567.1. Clade$\mathrm{V}$ of both MEPT and NJPT comprised with seventeen different Meloidogyne spp., were further differentiated into two subclades. Sub-clade-1 comprised of eleven distantly related Meloidogyne spp. (JX465564.1, KC342236.1, JX465565.1, JX465566.1， JX465576.1， JX465574.1， JX465578.1， JX465570.1, JX465569.1, KP179223.1 and KP179229.1), and Sub-clade-2 comprised of six closely related Meloidogyne spp. (JX885741.1， LC030354.1， LC030352.1， JX465561.1， JX465562.1 and KF482363.1). Interestingly, both the MEPT and MJPT analysis revealed that most virulent strains of the world M. javanica (KC953091.1, U96305.1), M. arenaria (U96301.1, LC030350.1, AY438554.1, AF387092.1, LC030351.1, LC030355.1) and M. incognita (JQ405212.1, KJ641591.1, AY438556.1, KC464469.1,

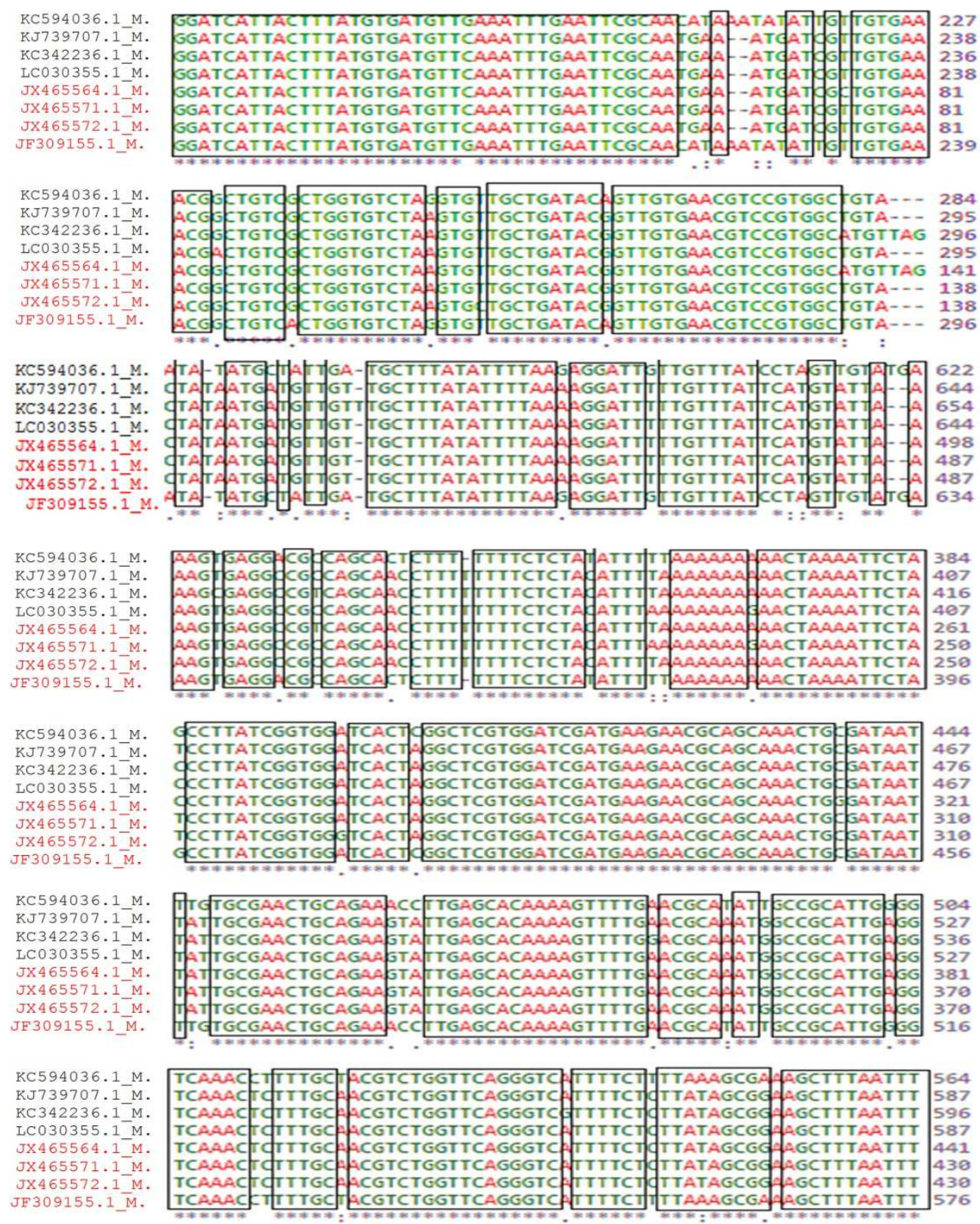

Figure 1. Alignment result showing conserved region that are identical in both highly virulent (M. arenaria and M. incognita) nematodes and some other nematodes species (M. enterolobii_1, M. sp._Mi_c3a, M. sp_Mj_cla, M._sp._Mj_c3a). 


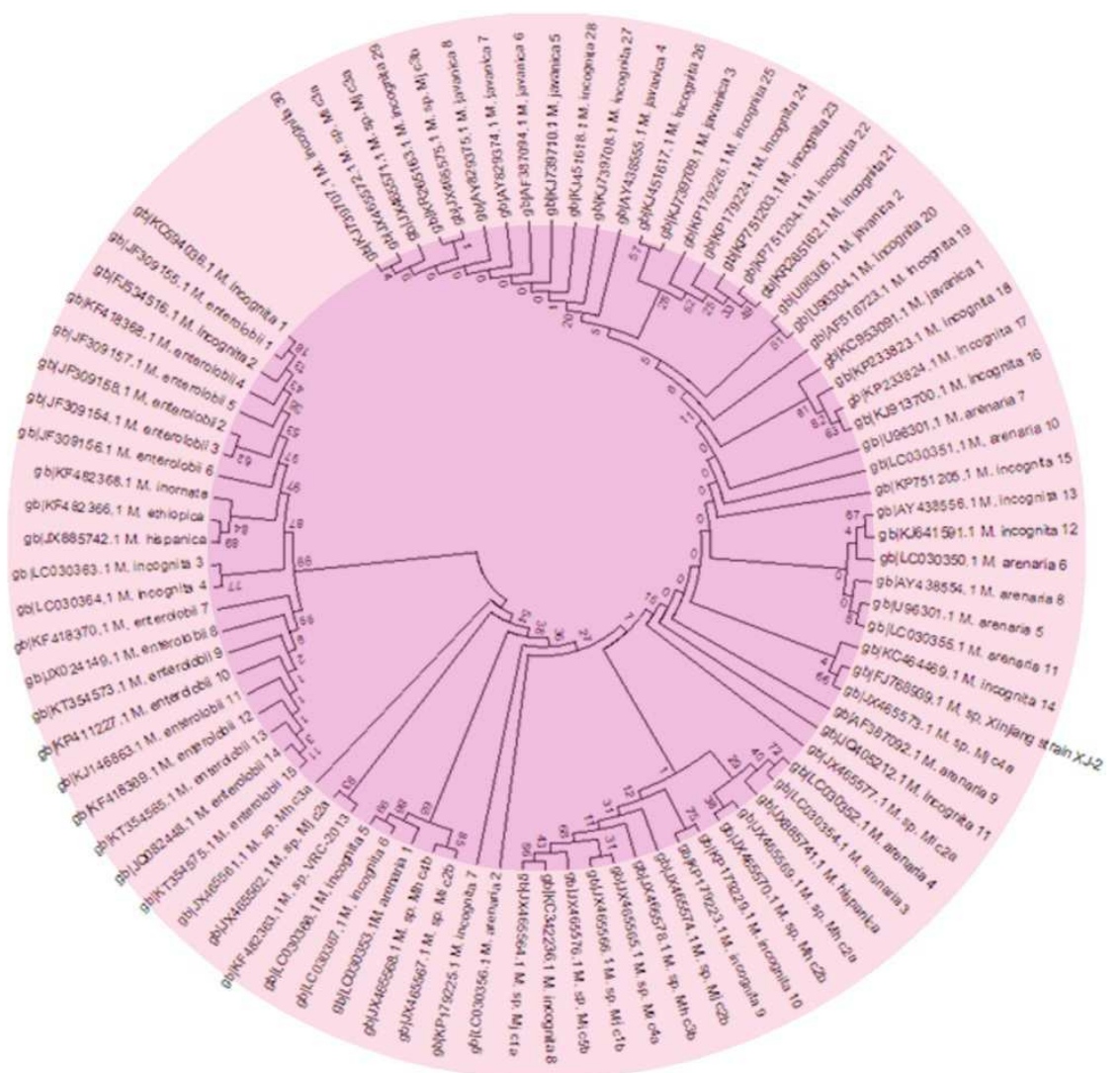

Figure 2. The phylogenetic tree of Meloidogyne species was inferred using the Neighbor-Joining method.

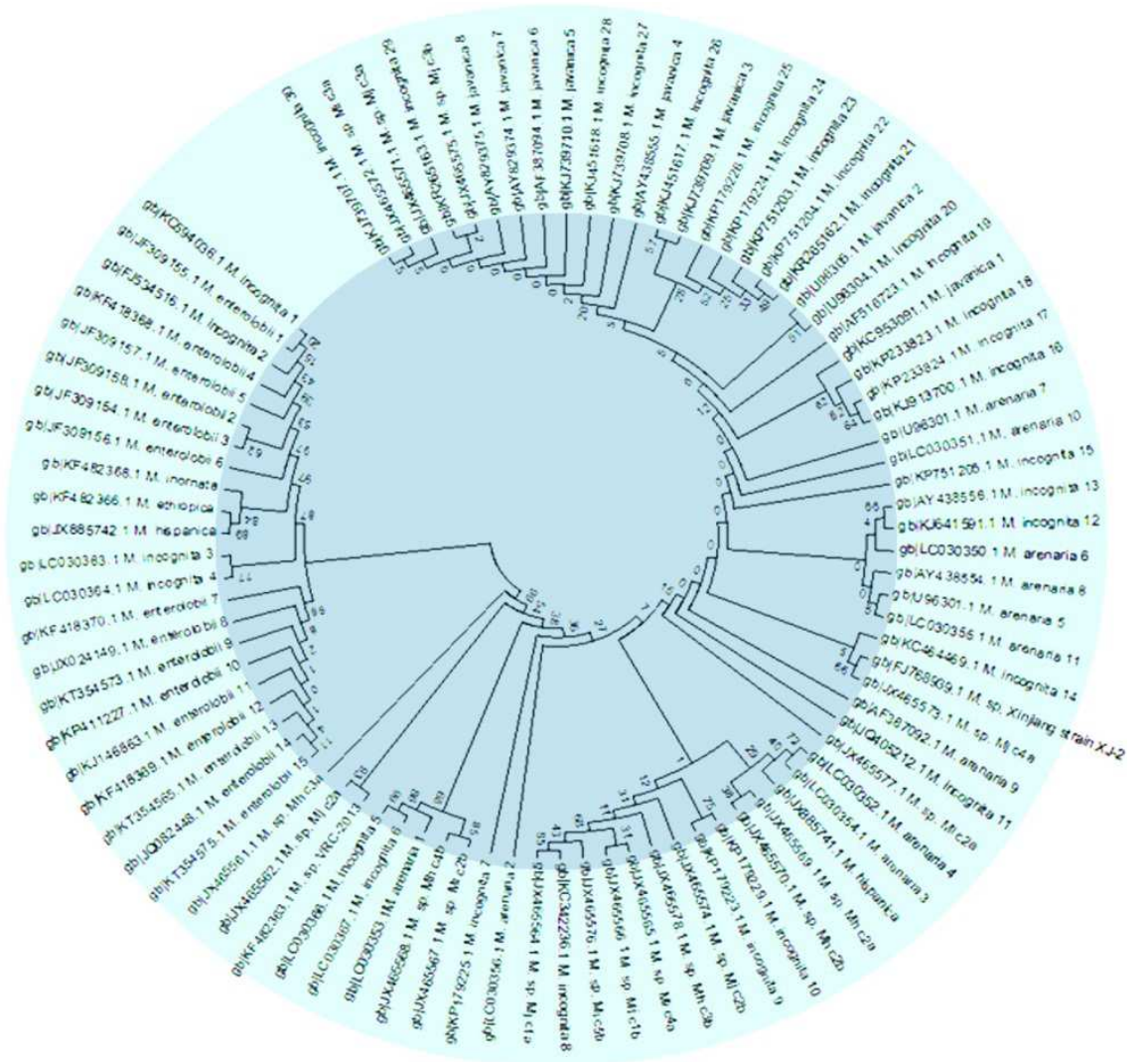

Figure 3. The phylogenetic tree of Meloidogyne species was inferred using the Minimum Evolution method.

KP751205.1, KJ913700.1, KP233824.1, KP233823.1, AF516723.1, U96304.1) were clustered in Clade-VI. 
Previous study supported the close relationships between the three major ameiotic species; M. arenaria, M. javanica and M. incognita. Major clade-VII differentiated into two subclades in both the case of MEPT and NJPT. Sub-clade-1 represented the seven closely related strains of $M$. javanica (KJ739709.1) and M. incognita (KR265162.1, KP751204.1,
KP751203.1, KP179224.1, KP179226.1, KJ451617.1) and sub-clade-2 stand for eleven different nematodes of $M$. Javanica (AY438555.1， KJ739710.1， AF387094.1, AY823974.1, AY823975.1) and M. Incognita (KJ739708.1, KJ451618.1， KR265163.1， KJ739707.1) except (JX465575.1, JX465572.1) [8].

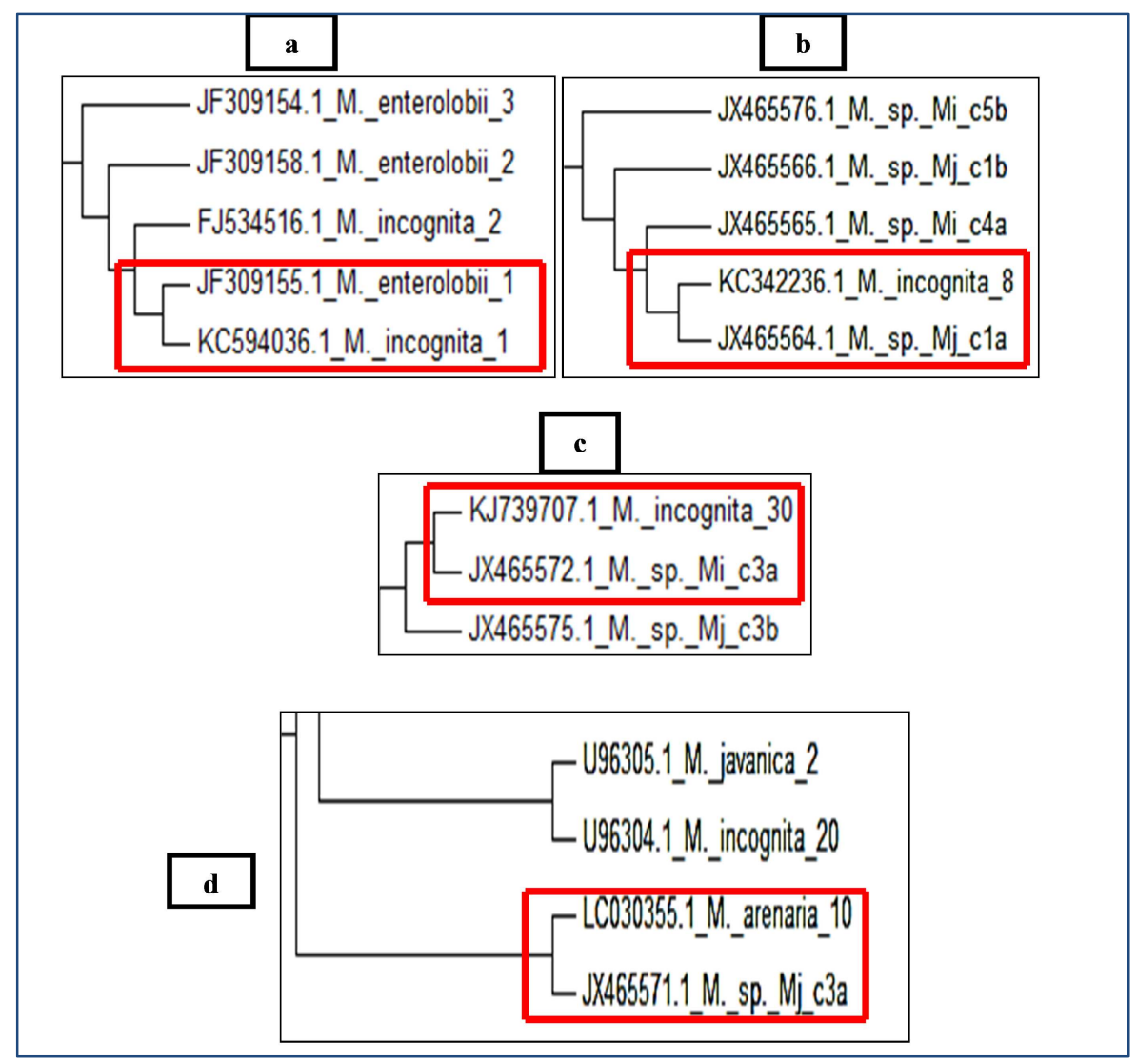

Figure 4. M. enterolobii_1, M. sp_Mj_cla, M. sp._Mi_c3a were sharing the same clade with different strains of M. incognita (tagged by a, $b, c$ respectively) and M._sp._Mj_c3a shared the same clade with M. arenaria (tagged byd) in both Neighbor-Joining method and Evolution method.

Phylogenetic tree showed that $M$. hispanicais distantly related with $M$. incognita, M. arenaria, M. Enterolobii and some other root-knot nematode species [34]. All parasitic nematodes were originally evolved from free living nematodes [35-38]. The adoption of parasitism in nematodes probably required either the adaptation of genes present in their free-living ancestors or horizontal gene transfer from bacteria and/or fungus in their environment [39-41]. Thus, there is a good chance that the existing non-virulent species of Meloidogyne can become virulent due to single or integrated aforementioned factors. Therfore, evolutionally emerging strains of existing virulent species $M$. javanica, $M$. arenaria and $M$. incognita along with the potential nematodes $M$. enterolobii_1, M. sp._Mi_c3a, $M$. $s p \_M j \_l a$ that shared the same clade with $M$. incognita and $M$._sp._Mj_c3a are evolutionally closely related with $M$. arenaria (Figure 4) could become a great challenge for world agriculture.

\section{Conclusion}

Nematodes, the most widespread organisms on Earth are capable of colonizing any ecosystem, including extreme environments, such as deserts, hot spring waters, arctic lands and polar seas and are also making impact in agriculture. The unseen enemy of world agriculture rapidly becomes more virulent and in a little while can be responsible for world food crisis. Immediately measures should be taken against the newly emerging strains and potential nematodes species. The study presenting some of such potential nematodes $(M$. enterolobii_1, M. sp._Mi_c3a, M. sp_Mj_cla, M._sp._Mj_c3a) based on the genetic as well as evolutionary relationship with existing well recognized virulent nematodes $M$. javanica, $M$. arenaria and $M$. incognita. Further study is needed to more clearly understand the molecular relationship among them that will be beneficial to take more develop control measures.

\section{References}

[1] C. Wang, S. Lower, V. P. Thomas and V. M. Williamson, "Root-knot nematodes exhibit strain-specific clumping behavior that is inherited as a simple genetic trait," PloS One, vol. 5 (12), pp. e15148, December 2010. 
[2] Mantelin, S., Bellafiore, S. and Kyndt, T., "Meloidogyne graminicola: a major threat to rice agriculture," Molecular plant pathology, 18 (1), p. 3, 2017.

[3] Archidona-Yuste, A., Cantalapiedra-Navarrete, C., Liébanas, G., Rapoport, H. F., Castillo, P. and Palomares-Rius, J. E., "Diversity of root-knot nematodes of the genus Meloidogyne Göeldi, 1892 (Nematoda: Meloidogynidae) associated with olive plants and environmental cues regarding their distribution in southern Spain" PloS one, 13 (6), p. e0198236, 2018.

[4] J. N. Sasser, "Economic importance of Meloidogyne in tropical countries," Root-knot nematodes, pp. 359-374, 1979.

[5] R. A. Sikora and E. Fernandez, "Nematode parasites of vegetables," Plant Parasitic Nematodes in Subtropical and Tropical Agriculture, pp. 319, 2005.

[6] W. M. Wesemael, N. Viaene and M. Moens, "Root-knot nematodes (Meloidogyne spp.) in Europe," Nematology, vol. 13 (1), pp. 3-16, January 2011.

[7] Janssen, T., Karssen, G., Topalović, O., Coyne, D. and Bert, W., "Integrative taxonomy of root-knot nematodes reveals multiple independent origins of mitotic parthenogenesis" PloS one, 12 (3), p. e 0172190, 2017.

[8] Janssen, T., Karssen, G., Verhaeven, M., Coyne, D. and Bert, W., "Mitochondrial coding genome analysis of tropical rootknot nematodes (Meloidogyne) supports haplotype based diagnostics and reveals evidence of recent reticulate evolution" Scientific Reports, 6, p. 22591, 2016.

[9] Hamza, M. A., Ali, N., Tavoillot, J., Fossati-Gaschignard, O., Boubaker, H., El Mousadik, A. and Mateille, T., "Diversity of root-knot nematodes in Moroccan olive nurseries and orchards does Meloidogyne javanica disperse according to invasion processes?" BMC ecology, 17 (1), p. 41, 2017.

[10] M. Moens, R. N. Perry and J. L. Starr, "Meloidogyne speciesa diverse group of novel and important plant parasites," Rootknot nematodes, vol. 1, pp. 483, 2009.

[11] A. C. Triantaphyllou, "Cytogenetics, cytotaxonomy and phylogeny of root-knot nematodes," 1985.

[12] Sun, L., Zhuo, K., Lin, B., Wang, H. and Liao, J., "The complete mitochondrial genome of Meloidogyne graminicola (Tylenchina): a unique gene arrangement and its phylogenetic implications" Plos one, 9 (6), p. e98558, 2014.

[13] T. Powers, "Nematode molecular diagnostics: From bands to barcodes," Annu. Rev. Phytopathol, vol. 42, pp. 367-383, September 2004

[14] I. T. De Ley, P. De Ley, A. Vierstraete, G. Karssen, M. Moens and J. Vanfleteren, "Phylogenetic analyses of Meloidogyne small subunit rDNA," Journal of Nematology, vol. 34 (4), pp. 319, December 2002

[15] R. Nakacwa, A. Kiggundu, H. Talwana, J. Namaganda, C. Lilley, W. Tushemereirwe and H. Atkinson, "Nematode 18S rRNA gene is a reliable tool for environmental biosafety assessment of transgenic banana in confined field trials," Transgenic research, vol. 22 (5), pp. 1003-1010, October 2013

[16] J. Brito, T. O. Powers, P. G. Mullin, R. N. Inserra, D. W. Dickson, "Morphological and molecular characterization of Meloidogyne mayaguensis isolates from Florida," Journal of Nematology, vol. 36 (3), pp. 232, September 2004.
[17] N. Vovlas, D. Mifsud, B. B. Landa and P. Castillo, "Pathogenicity of the root-knot nematode Meloidogyne javanica on potato," Plant Pathology, vol. 54 (5), pp. 657-664, October 2005.

[18] Ali, M. A., Azeem, F., Abbas, A., Joyia, F. A., Li, H. and Dababat, A. A., "Transgenic strategies for enhancement of nematode resistance in plants" Frontiers in plant science, 8 , p. $750,2017$.

[19] Sultana, T., Kim, J., Lee, S. H., Han, H., Kim, S., Min, G. S., Nadler, S. A. and Park, J. K., "Comparative analysis of complete mitochondrial genome sequences confirms independent origins of plant-parasitic nematodes" $B M C$ evolutionary biology, 13 (1), p. 12, 2013.

[20] E. K. Tomaszewski, M. A. M. Khalil, A. A. Er- Deeb, T. O. Powers, J. L. Starr, "Meloidogyne javanica parasitic on peanut," Journal of Nematology, vol. 26 (4), pp. 436-441, December 1994.

[21] F. Lamberti and R. C. Baines, "Pathogenicity of four species of Meloidogyne on three varieties of olive trees," Journal of Nematology, vol. 1, pp. 111-115, April 1969.

[22] N. Sasanelli, G. Fontanazza, F. D. Lamberti, T. D’ Addabbo, M. Patumi and G. Vergari, "Reaction of olive cultivars to Meloidogyne species," Nematol. Mediterr., vol. 25, pp. 183190, December 1997.

[23] I. A. Udo, M. I. Uguru and R. O. Ogbuji, "Pathogenicity of Meloidogyne incognita race 1 on tomato as influenced by different arbuscular mycorrhizal fungi and bioformulated Paecilomyces lilacinus in dysteric cambisol soil," Journal of Plant Protection Research, vol. 53 (1), pp. 71-78, January 2013.

[24] C. M., Maleita, M. J. Simões, C. Egas, R. H. C. Curtis \& I. M. De O. Abrantes. Biometrical, biochemical, and molecular diagnosis of Portuguese Meloidogyne hispanica isolates. Plant Disease, 96 (6): 865-874, 2012.

[25] Y. F. Wang, S. Xiao, Y. K. Huang, X. Zhou, S. S. Zhang and G. K. Liu, "First Report of Meloidogyne enterolobii on carrot in China," APS journal, vol. 98 (7), pp. 1019, June 2014.

[26] Hasan, M., Hakim, A., Iqbal, A., Bhuiyan, F. R., Begum, M. K., Sharmin, S. and Abir, R. A.,"Computational study and homology modeling of phenol hydroxylase: key enzyme for phenol degradation" Int J Comput Bioinfo In Silico Model. 2015b, 4 (4), pp. 691-698, 2015.

[27] Hasan, M., Ghosh, P. P., Azim, K. F., Mukta, S., Abir, R. A., Nahar, J. and Khan, M. M. H., "Reverse vaccinology approach to design a novel multi-epitope subunit vaccine against avian influenza A (H7N9) virus. Microbial pathogenesis, 130, pp. 19-37, 2019.

[28] Das, K., Chakraborty, S., Hasan, M. and Shovo, A. M., "In silico analysis to elect superior bacterial alkaline protease for detergent and leather industries" Journal of Advances In Biotechnology, 5 (3), pp. 685-698, 2016.

[29] K. Tamura, G. Stecher, D. Peterson, A. Filipski and S. Kumar, "MEGA6: molecular evolutionary genetics analysis version 6 . 0," Molecular Biology and Evolution, vol. 30 (12), pp. 2725 2729, October 2013

[30] K. Tamura, M. Nei and S. Kumar, "Prospects for inferring very large phylogenies by using the neighbor-joining method," Proceedings of the National Academy of Sciences (USA), vol. 101, pp. 11030-11035, July 2004. 
[31] J. N. Sasser, "Plant-parasitic nematodes: the farmer's hidden enemy. Plant-parasitic nematodes: the farmer's hidden enemy," 1989.

[32] Tian, B. Y., Cao, Y. and Zhang, K. Q., "Metagenomic insights into communities, functions of endophytes, and their associates with infection by root-knot nematode, Meloidogyne incognita, in tomato roots" Scientific reports, 5, p. 17087, 2015 .

[33] Warmerdam, S., Sterken, M. G., van Schaik, C., Oortwijn, M. E., Sukarta, O. C., Lozano-Torres, J. L., Dicke, M., Helder, J., Kammenga, J. E., Goverse, A. and Bakker, J., "Genome-wide association mapping of the architecture of susceptibility to the root-knot nematode Meloidogyne incognita in Arabidopsis thaliana”. New Phytologist, 218 (2), pp. 724-737, 2018.

[34] B. B. Landa, J. E. P. Rius, N. Vovlas, R. M. Carneiro, C. M. Maleita, I. M. de O. Abrantes and P. Castillo, "Molecular characterization of Meloidogyne hispanica (Nematoda, Meloidogynidae) by Phylogenetic Analysis of Genes Within the rDNA in Meloidogyne spp," Plant Disease, vol. 92 (7), pp. 1104-1110, July 2008.

[35] M. L. Blaxter, P. De Ley, J. R. Garey, L. X. Liu, P. Scheldeman, A. Vierstraete, J. R. Vanfleteren, L. Y. Mackey, M. Dorris, L. M. Frisse and J. T. Vida, "A molecular evolutionary framework for the phylum Nematoda," Nature, vol. 392 (6671), pp. 71-75, March 1998.
[36] M. L. Blaxter, "Nematoda: genes, genomes and the evolution of parasitism," Advances in Parasitology, vol. 54, pp. 101-195, January 2003.

[37] Teillet, A., Dybal, K., Kerry, B. R., Miller, A. J., Curtis, R. H. and Hedden, P., "Transcriptional changes of the root-knot nematode Meloidogyne incognita in response to Arabidopsis thaliana root signals". PloS one, 8 (4), p. e61259, 2013.

[38] Tao, Y., Xu, C., Yuan, C., Wang, H., Lin, B., Zhuo, K. and Liao, J., "Meloidogyne aberrans sp. nov. (Nematoda: Meloidogynidae), a new root-knot nematode parasitizing kiwifruit in China". PloS one, 12 (8), p. e0182627, 2017.

[39] K. Kiontke, N. P. Gavin, Y. Raynes, C. Roehrig, F. Piano and D. H. Fitch, "Caenorhabditis phylogeny predicts convergence of hermaphroditism and extensive intron loss," Proceedings of the National Academy of Sciences of the United States of America, vol. 101 (24), pp. 9003-9008, June 2004.

[40] M. Mitreva, G. Smant and J. Helder, "Role of horizontal gene transfer in the evolution of plant parasitism among nematodes," Methods Mol. Biol, vol. 532, pp. 517-535, 2009.

[41] E. H., Scholl, J. L., Thorne, J. P. McCarterand, D. M., Bird, (2003). Horizontally transferred genes in plant-parasitic nematodes: a high-throughput genomic approach. Genome Biol, 4 (6), p. R39. 